\title{
The general notion of descent in coarse geometry
}

\author{
PAUl D MitCHENER
}

In this article, we introduce the notion of a functor on coarse spaces being coarsely excisive - a coarse analogue of the notion of a functor on topological spaces being excisive. Further, taking cones, a coarsely excisive functor yields a topologically excisive functor, and for coarse topological spaces there is an associated coarse assembly map from the topologically excisive functor to the coarsely excisive functor.

We conjecture that this coarse assembly map is an isomorphism for uniformly contractible spaces with bounded geometry, and show that the coarse isomorphism conjecture, along with some mild technical conditions, implies that a corresponding equivariant assembly map is injective. Particular instances of this equivariant assembly map are the maps in the Farrell-Jones conjecture, and in the Baum-Connes conjecture.

55N20; 20F05

\section{Introduction}

In coarse geometry the term descent usually refers to the fact that the coarse BaumConnes conjecture for a group $G$ equipped with a word length metric, along with a few fairly mild conditions on the space $E G$, implies rational injectivity of the analytic Novikov assembly map described by Kasparov [18; 19]. We refer the reader to Higson and Roe [14; 15] and Roe [34] for details of the argument. This descent principle has been generalised to nonmetric coarse structures, such as those arising from compactifications, as described by Higson, Pedersen and Roe [13].

There are similar coarse notions for assembly maps in algebraic $K$ - and $L$-theory arising from controlled topology. For example, the descent result involving coarse structures arising from compactifications was inspired by the work in controlled algebra of Carlsson and Pedersen [6]. A very strong parallel with methods in the coarse Baum-Connes conjecture can be found in [2], where methods used to prove the coarse Baum-Connes conjecture for spaces of finite asymptotic dimension in Wright [41] and $\mathrm{Yu}$ [42] are used to show that the algebraic $K$-theory assembly map (see Bökstedt, Hsiang and Madsen [5] and Loday [21]) is injective for groups with finite asymptotic dimension. 
It is our purpose in this article to give a general theory of coarse assembly maps and descent techniques that incorporates all of the above. We define a general notion of a coarse assembly map. Our main result essentially shows that the coarse assembly map being an isomorphism, along with certain mild technical conditions, implies that the corresponding equivariant coarse assembly map is injective.

Cases of the equivariant coarse assembly map include the analytic Novikov assembly map, the Baum-Connes assembly map (see Baum, Connes and Higson [4]), and the Farrell-Jones assembly map in algebraic $K$-theory (see Farrell and Jones [9]).

In principle, geometric arguments used to prove the coarse Baum-Connes conjecture can be adapted to the new abstract framework. For example, the results of the author in [27] immediately tell us that every coarse assembly map is an isomorphism for finite coarse cellular complexes.

Wright's proof of the coarse Baum-Connes conjecture for spaces of finite asymptotic dimension in [41] is also purely geometric, and should extend to the present set-up. Finally, there is some hope of applying these ideas to spaces of finite decomposition complexity, as described by Guentner, Tessera and $\mathrm{Yu}$ [11].

The author wishes to take this opportunity to thank the referee of this paper for his insight and evident hard work.

\section{Coarse spaces}

Recall that a coarse space is a set $X$ equipped with a number of distinguished subsets of the product $X \times X$ called controlled sets.

The collection of controlled sets is required to be closed under finite unions, taking subsets, reflections in the diagonal of $X \times X$, and composition in the sense that we define

$$
M_{1} M_{2}=\left\{(x, z) \in X \times X \mid(x, y) \in M_{1},(y, z) \in M_{2} \text { for some } y \in X\right\}
$$

for controlled sets $M_{1}$ and $M_{2}$.

We further require the diagonal, $\Delta_{X}=\{(x, x) \mid x \in X\}$, to be controlled, ${ }^{1}$ and the union of all controlled sets to be the entire space $X \times X$. We refer the reader to Roe [36], for example, for further details.

\footnotetext{
${ }^{1}$ The assumption that the diagonal is a controlled set is sometimes dropped; a coarse structure where this axiom does not hold is termed nonunital. Many of our definitions should be modified somewhat in the nonunital case; see Luu [22].
} 
Following Wright [40], we call a coarse topological space $X$ separable if there is a countable locally finite cover $\left(U_{i}\right)$ of $X$ with $\bigcup_{i} U_{i} \times U_{i}$ controlled.

Given a controlled set $M \subseteq X \times X$, and a subset $S \subseteq X$, we write

$$
M[S]=\{y \in X \mid(x, y) \in M \text { for some } x \in S\} .
$$

For a point $x \in X$, we write $M(x)=M[\{x\}]$.

If $X$ is a coarse space, and $f, g: S \rightarrow X$ are maps into $X$, the maps $f$ and $g$ are termed close or coarsely equivalent if the set $\{(f(s), g(s)) \mid s \in S\}$ is controlled. We call a subset $B \subseteq X$ bounded if the inclusion $B \hookrightarrow X$ is close to a constant map, or equivalently $B=M(x)$ for some controlled set $M$ and some point $x \in X$.

The following definition comes from Higson, Pedersen and Roe [13].

Definition 2.1 Let $X$ be a Hausdorff space. A coarse structure on $X$ is said to be compatible with the topology if every controlled set is contained in an open controlled set, and the closure of any bounded set is compact.

We call a Hausdorff space equipped with a coarse structure that is compatible with the topology a coarse topological space.

Note that any coarse topological space is locally compact. In such a space, the bounded sets are precisely those which are precompact. It also follows from the definition that any precompact subset of the product of the space with itself is a controlled set, and the closure of any controlled set is controlled.

Example 2.2 If $X$ is a proper metric space, the bounded coarse structure is the unital coarse structure formed by defining the controlled sets to be subsets of neighbourhoods of the diagonal:

$$
N_{R}=\{(x, y) \in X \times X \mid d(x, y)<R\}
$$

The bounded sets are simply those which are bounded with respect to the metric. If we give the metric space $X$ the bounded coarse structure, it is a coarse topological space.

The following example is a generalisation of the continuously controlled coarse structure arising from a compactification, as found for instance in [13].

Example 2.3 Let $X$ be a coarse topological space, and suppose that $X$ is contained in a Hausdorff topological space $\bar{X}$ as a topologically dense subset. Call the coarse structure already defined on the space $X$ the ambient coarse structure. 
Write $\partial X=\bar{X} \backslash X$. Call an open subset $M \subseteq X \times X$ strongly controlled if:

- The set $M$ is controlled with respect to the ambient coarse structure on $X$.

- Let $\bar{M}$ be the closure of the set $M$ in the space $\bar{X}$. Then

$$
\bar{M} \cap(\bar{X} \times \partial X \cup \partial X \times \bar{X}) \subseteq \Delta_{\partial X} .
$$

Then we define the continuously controlled coarse structure with respect to $\bar{X}$ by saying that the controlled sets are composites of subsets of strongly controlled open sets.

We write $X$ to denote the space $X$ with its ambient coarse structure, and $X^{\text {cc }}$ to denote the space $X$ with the new continuously controlled coarse structure.

Proposition 2.4 The space $X^{\mathrm{cc}}$ is a coarse topological space. Further, if $B \subseteq X^{c c}$ is bounded, then $\bar{B} \cap \partial X=\varnothing$.

Proof By construction, we have a coarse structure where every controlled set is a subset of an open controlled set.

Let $B \subseteq X^{\mathrm{cc}}$ be bounded. Then the set $B$ is also bounded with respect to the ambient coarse structure on the space $X$. Since the ambient coarse structure is compatible with the topology, the closure of the set $B$ in the space $X$ is compact. Thus the continuously controlled coarse structure is compatible with the topology.

Since the closure of the set $B$ is compact in the space $X$, it is also closed in the space $\bar{X}$. We see $\bar{B} \subseteq X$, so $\bar{B} \cap \partial X=\varnothing$.

Let $X$ and $Y$ be coarse spaces. Then a map $f: X \rightarrow Y$ is said to be controlled if for every controlled set $M \subseteq X \times X$, the image

$$
f[M]=\{(f(x), f(y)) \mid(x, y) \in M\}
$$

is a controlled set. A controlled map is called coarse if the inverse image of any bounded set is bounded.

We can form the category of all coarse spaces and coarse maps. We call this category the coarse category. We call a coarse map $f: X \rightarrow Y$ a coarse equivalence if there is a coarse map $g: Y \rightarrow X$ such that the composites $g \circ f$ and $f \circ g$ are close to the identities $1_{X}$ and $1_{Y}$ respectively.

Coarse spaces $X$ and $Y$ are said to be coarsely equivalent, and we write $X \sim Y$, if there is a coarse equivalence between them. 
Definition 2.5 Let $X$ and $Y$ be coarse spaces. Then we define the product, $X \times Y$ to be the Cartesian product of the sets $X$ and $Y$ equipped with the coarse structure defined by saying a subset $M \subseteq(X \times Y) \times(X \times Y)$ is controlled if it is the subset of a set of the form

$$
\left\{(u, v, x, y) \mid(u, x) \in M_{1},(v, y) \in M_{2}\right\}
$$

where $M_{1} \subseteq X \times X$ and $M_{2} \subseteq Y \times Y$ are controlled sets.

Note that the above product is not in general a product in the category-theoretic sense, since the projections onto the factors are not coarse maps.

Definition 2.6 Let $X$ be a coarse space, let $\sim$ be an equivalence relation on $X$, and let $X / \sim$ be the set of equivalence classes. Let $\pi: X \rightarrow X / \sim$ be the quotient map sending each point $x \in X$ to its equivalence class, $\pi(x)$.

We define the quotient coarse structure on $X / \sim$ by saying a subset $M \subseteq X / \sim \times X / \sim$ is controlled if and only if $M=\pi\left[M^{\prime}\right]$ for some controlled set $M^{\prime} \subseteq X \times X$.

Note that the quotient map $\pi: X \rightarrow X / \sim$ is not in general a coarse map.

Definition 2.7 Let $\left\{X_{i} \mid i \in I\right\}$ be a collection of coarse spaces. Then, as a set, the coarse disjoint union, $\bigvee_{i \in I} X_{i}$ is the disjoint union of the sets $X_{i}$.

A subset $M \subseteq\left(\bigvee_{i \in I} X_{i}\right) \times\left(\bigvee_{i \in I} X_{i}\right)$ is controlled if it is a subset of a union of the form

$$
\left(\bigcup_{i \in I} M_{i}\right) \cup\left(\bigcup_{i, j \in I} B_{i} \times B_{j}\right)
$$

where each set $M_{i} \subseteq X_{i} \times X_{i}$ is controlled, and $B_{j} \subseteq X_{j}$ is bounded.

For example, let $\mathbb{R}_{+}$be the space $[0, \infty)$ equipped with the bounded coarse structure arising from the usual metric. Then $\mathbb{R}_{+} \vee \mathbb{R}_{+} \sim \mathbb{R}$, where the real line, $\mathbb{R}$, is again given the bounded coarse structure.

\section{Coarse homotopy}

The following definition comes from [27].

Definition 3.1 Let $R$ be the topological space $[0, \infty)$ equipped with a coarse structure compatible with the topology. We call the space $R$ a generalised ray if the following conditions hold. 
- Let $M, N \subseteq R \times R$ be controlled sets. Then the sum

$$
M+N=\{(u+x, v+y) \mid(u, v) \in M,(x, y) \in N\}
$$

is controlled.

- Let $M \subseteq R \times R$ be a controlled set. Then the set

$$
M^{s}=\{(u, v) \in R \times R \mid x \leq u, v \leq y,(x, y) \in M\}
$$

is controlled.

- Let $M \subseteq R \times R$ be a controlled set, and $a \in R$. Then the set

$$
a+M=\{(a+x, a+y) \mid(x, y) \in M\}
$$

is controlled.

For example, the space $\mathbb{R}_{+}$(with the bounded coarse structure) is a generalised ray. The space $[0, \infty)$ equipped with the continuously controlled coarse structure arising from the one point compactification is also a generalised ray.

In order to look at the notion of homotopy for coarse spaces, we first consider cylinders. Our first definition is inspired by Section 3 of Dranishnikov [8].

Definition 3.2 Let $X$ be a coarse space equipped with a coarse map $p: X \rightarrow R$. Then we define the $p$-cylinder of $X$ :

$$
I_{p} X=\{(x, t) \in X \times R \mid t \leq p(x)+1\}
$$

We define coarse maps $i_{0}, i_{1}: X \rightarrow I_{p} X$ by the formulae $i_{0}(x)=(x, 0)$ and $i_{1}(x)=$ $(x, p(x)+1)$ respectively.

The following definition is inspired by Mitchener, Norouzizadeh and Schick [32].

Definition 3.3 Let $f_{0}, f_{1}: X \rightarrow Y$ be coarse maps. An elementary coarse homotopy between $f_{0}$ and $f_{1}$ is a coarse map $H: I_{p} X \rightarrow Y$ for some $p: X \rightarrow R$ such that $f_{0}=H \circ i_{0}$ and $f_{1}=H \circ i_{1}$.

More generally, we call the maps $f_{0}$ and $f_{1}$ coarsely homotopic if they can be linked by a chain of elementary coarse homotopies.

A coarse map $f: X \rightarrow Y$ is termed a coarse homotopy equivalence if there is a coarse map $g: Y \rightarrow X$ such that the compositions $g \circ f$ and $f \circ g$ are coarsely homotopic to the identities $1_{X}$ and $1_{Y}$ respectively. 
Example 3.4 Let $X$ and $Y$ be spaces, and let $p: X \rightarrow R$ be a coarse map. Consider two close coarse maps $f_{0}: X \rightarrow Y$ and $f_{1}: X \rightarrow Y$. Then we can define a coarse homotopy $H: I_{p} X \rightarrow Y$ between the maps $f_{0}$ and $f_{1}$ by the formula

$$
H(x, t)= \begin{cases}f_{0}(x) & x<1, \\ f_{1}(x) & x \geq 1 .\end{cases}
$$

Thus, close maps are also coarsely homotopic. In particular, any coarse equivalence is also a coarse homotopy equivalence.

\section{Coarse homology}

The following definition comes from Higson, Roe and $\mathrm{Yu}$ [16], where it is a condition for the existence of a coarse version of the Mayer-Vietoris sequence.

Definition 4.1 Let $X$ be a coarse space. Then we call a decomposition $X=A \cup B$ coarsely excisive if for every controlled set $m \subseteq X \times X$ there is a controlled set $M \subseteq X \times X$ such that $m(A) \cap m(B) \subseteq M(A \cap B)$.

The following definition is now a slight variant of the definition in [27].

Definition 4.2 A coarse homology theory is a collection of functors, $\left\{h_{n}^{\text {coarse }} \mid n \in \mathbb{Z}\right\}$, from the coarse category to the category of abelian groups such that the following axioms are satisfied:

- Let $X$ be a coarse space, and let $f, g: X \rightarrow Y$ be coarsely homotopic coarse maps. Then the induced homomorphisms $f_{*}, g_{*}: h_{*}^{\text {coarse }}(X) \rightarrow h_{*}^{\text {coarse }}(Y)$ are equal.

- Let $X$ be a coarse space, and let $X=A \cup B$ be a coarsely excisive decomposition. Let $i: A \cap B \hookrightarrow A, j: A \cap B \hookrightarrow B, k: A \hookrightarrow X$, and $l: B \hookrightarrow X$ be the associated inclusion maps. Then there are natural maps $\partial: h_{n}^{\text {coarse }}(X) \rightarrow h_{n-1}^{\text {coarse }}(X)$ such that we have a long exact sequence:

$\rightarrow h_{n}^{\text {coarse }}(A \cap B) \stackrel{\left(i_{*},-j_{*}\right)}{\longrightarrow} h_{n}^{\text {coarse }}(A) \oplus h_{n}^{\text {coarse }}(B) \stackrel{k_{*}+l_{*}}{\longrightarrow} h_{n}^{\text {coarse }}(X) \stackrel{\partial}{\rightarrow} h_{n-1}^{\text {coarse }}(A \cap B) \rightarrow$

The above long exact sequence is called a coarse Mayer-Vietoris sequence.

The above axioms are a coarse variant of the Eilenberg-Steenrod axioms used to define a generalised homology theory in the world of topological spaces.

The following definition essentially comes from $[13 ; 34]$. 
Definition 4.3 We call a coarse space $X$ flasque if there is a map $t: X \rightarrow X$ such that:

- Let $B \subseteq X$ be bounded. Then there exists $N \in \mathbb{N}$ such that $t^{n}[X] \cap B=\varnothing$ for all $n \geq N$.

- Let $M \subseteq X \times X$ be controlled. Then the union $\bigcup_{n \in \mathbb{N}} t^{n}[M]$ is controlled.

- The map $t$ is close to the identity map.

In the above definition, the map $t$ is controlled by the second property, but is not necessarily coarse.

Observe that any generalised ray is flasque; the relevant map $t: R \rightarrow R$ is defined by the formula $t(s)=s+1$.

Definition 4.4 We call a functor, $E$, from the coarse category to the category of spectra coarsely excisive if the following conditions hold.

- The spectrum $E(X)$ is weakly contractible whenever the coarse space $X$ is flasque.

- The functor $E$ takes coarse homotopy equivalences to weak homotopy equivalences of spectra.

- For a coarsely excisive decomposition $X=A \cup B$ we have a homotopy push-out diagram

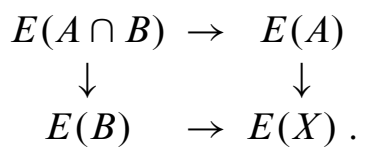

The following is immediate.

Proposition 4.5 Let $E$ be a coarsely excisive functor. Then the sequence of functors $X \mapsto \pi_{n} E(X)$ forms a coarse homology theory.

Definition 4.6 Let $X$ be a compact Hausdorff space. Then we define the open cone to be the space

$$
\mathcal{O} X=\frac{X \times[0,1)}{X \times\{0\}}
$$

equipped with the continuously controlled coarse structure arising from the compactification

$$
\frac{X \times[0,1]}{X \times\{0\}}
$$


Suppose that $X$ is a subset of the unit sphere of some Hilbert space, $H$. Let $\varphi:[0,1) \rightarrow$ $[0, \infty)$ be a homeomorphism. Then we have an induced map $\varphi_{*}: \mathcal{O} X \rightarrow H$ defined by writing

$$
\varphi_{*}(x, t)=\varphi(t) x
$$

and we define $\mathcal{O}_{\varphi} X$ to be the image of the above map $\varphi_{*}$, equipped with the bounded coarse structure arising from the metric of the Hilbert space $H$.

Proposition 6.2.1 of [15] tells us the following.

Proposition 4.7 Let $X$ be a compact subset of the unit sphere of a Hilbert space $H$.

- Let $\varphi:[0,1) \rightarrow[0, \infty)$ be a homeomorphism. Then every controlled set for the space $\mathcal{O}_{\varphi} X$ is also controlled for the space $\mathcal{O} X$.

- Let $M$ be a controlled set for the space $\mathcal{O} X$. Then there is a homeomorphism $\varphi:[0,1) \rightarrow[0, \infty)$ such that the set $M$ is controlled for the space $\mathcal{O}_{\varphi} X$.

The idea of a functor being coarsely excisive is an analogue of the following notion from Weiss and Williams [39].

Definition 4.8 Let $\mathcal{C}$ be the category of topological spaces that are homotopy equivalent to $\mathrm{CW}$-complexes. We call a functor, $F$, from the category $\mathcal{C}$ to the category of spectra excisive if it takes homotopy equivalences of spaces to weak homotopy equivalences of spectra, up to homotopy takes disjoint unions of topological spaces to coproducts in the category of spectra, and given a space $X=U \cup V$, where $U$ and $V$ are open sets we have a homotopy push-out diagram

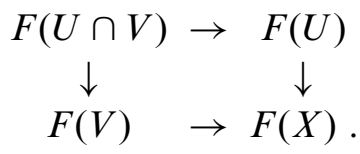

Given an excisive functor $F$, the sequence of functors $X \mapsto \pi_{n} F(X)$ forms a generalized homology theory.

Theorem 4.9 Let $E$ be a coarsely excisive functor. Then the assignment $X \mapsto E(\mathcal{O} X)$ defines an excisive functor on the category of compact Hausdorff spaces homotopy equivalent to $\mathrm{CW}$-complexes.

Proof Let $f: X \rightarrow Y$ be a continuous map. Then the obvious induced map $f_{*}: \mathcal{O} X \rightarrow$ $\mathcal{O} Y$ is coarse. 
The space $[0,1)$, equipped with the continuously controlled coarse structure arising from the one point compactification, can be considered to be a generalised ray. We have a coarse map $p: \mathcal{O} X \rightarrow[0,1]$ defined by the formula $p[(x, t)]=t$.

Consider a homotopy $F: X \times[0,1] \rightarrow Y$. Then the induced map is a coarse homotopy $F: I_{p} \mathcal{O} X \rightarrow Y$. Thus, given a homotopy equivalence $f: X \rightarrow Y$, we have an induced homotopy equivalence $f_{*}: E(\mathcal{O} X) \rightarrow E(\mathcal{O} Y)$.

Suppose we have a decomposition $X=U \cup V$, where $U$ and $V$ are open subsets. Embed $X$ in the unit sphere of a Hilbert space, $H$, and choose a homeomorphism $\varphi:[0,1) \rightarrow[0, \infty)$. Since the space $X$ is precompact, and the subsets $U$ and $V$ are open, for any $R>0$ we can find $S>0$ such that $N_{R}\left[\mathcal{O}_{\varphi} U\right] \cap N_{R}\left[\mathcal{O}_{\varphi} V\right] \subseteq N_{S}\left[\mathcal{O}_{\varphi}(U \cap V)\right]$.

It follows that the decomposition $\mathcal{O}_{\varphi} X=\mathcal{O}_{\varphi} U \cup \mathcal{O}_{\varphi} V$ is coarsely excisive. It follows by Proposition 4.7 that the decomposition $\mathcal{O} X=\mathcal{O} U \cup \mathcal{O} V$ is also coarsely excisive. It follows that we have a homotopy push-out square

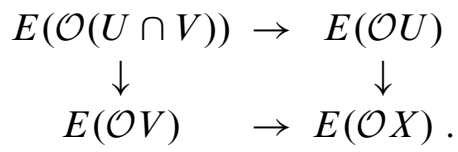

The result now follows.

\section{Coarse assembly}

Let $X$ be a coarse topological space. Then we define the open square to be the space $\mathcal{S} X=X \times[0,1)$ equipped with the continuously controlled coarse structure arising by considering $\mathcal{S} X$ as a dense subspace of the topological space $X \times[0,1]$.

We define the closed cone to be the quotient coarse space $\mathcal{C} X=X \times[0,1] / X \times\{0\}$. Note that there is no element of continuous control in the definition of the coarse structure on the closed cone; the coarse structure comes from the ambient coarse structure on the space $X$, and taking products and quotients.

Proposition 5.1 Let $X$ be a coarse topological space. Then the open square $\mathcal{S} X$ is flasque.

Proof Define a map $\alpha:[0,1) \rightarrow[0,1)$ by the formula

$$
\alpha(s)=\frac{1}{2-s} .
$$


Then the map $\alpha$ is a continuous monotonic increasing map, and for any point $s_{0} \in$ $[0,1)$, if we define the sequence $\left(s_{n}\right)$ iteratively by the formula $s_{n+1}=\alpha\left(s_{n}\right)$, then $\lim _{n \rightarrow \infty} s_{n}=1$.

Define a map $t: \mathcal{S} X \rightarrow \mathcal{S} X$ by the formula

$$
t(x, s)=(x, \alpha(s)) .
$$

Now, let $M \subseteq \mathcal{S} X \times \mathcal{S} X$ be strongly controlled. Then, looking at the ambient coarse structure, we have

$$
M \subseteq M_{X} \times[0,1) \times[0,1)
$$

where $M_{X} \subseteq X \times X$ is controlled. Here we are indulging in some slight abuse of notation involving the order of our factors.

Further, $\quad \bar{M} \cap((X \times[0,1] \times X \times\{1\}) \cup(X \times\{1\} \times X \times[0,1])) \subseteq \Delta_{X \times\{1\}}$.

It follows that $\quad t[M] \subseteq M_{X} \times[0,1) \times[0,1)$

and

$$
t[\bar{M}] \cap((X \times[0,1] \times X \times\{1\}) \cup(X \times\{1\} \times X \times[0,1])) \subseteq \Delta_{X \times\{1\}} .
$$

Iterating, we see that $\cup_{n \in \mathbb{N}} t^{n}[M]$ is controlled with respect to the continuously controlled coarse structure on the space $\mathcal{S} X$. In particular, it follows that the map $t$ is coarse.

Let

$$
M=\{((x, s),(x, \alpha(s))) \mid x \in X, s \in[0,1)\} .
$$

Then the set $M$ is certainly controlled with respect to the ambient coarse structure on $X$.

Let $\left(s_{n}\right)$ be a sequence in the space $[0,1)$. Observe that $s_{n} \rightarrow 1$ as $n \rightarrow \infty$ if and only if $\alpha\left(s_{n}\right) \rightarrow 1$ as $n \rightarrow \infty$. Hence

$$
\begin{aligned}
\bar{M} \cap((X \times[0,1] \times X \times\{1\}) \cup(X \times\{1\} \times(X \times[0,1])) & =\{((x, 1),(x, 1)) \mid x \in X\} \\
& =\Delta_{X \times\{1\} .}
\end{aligned}
$$

We see that the set $M$ is controlled with respect to the continuously controlled coarse structure on the space $\mathcal{S} X$. Hence the map $t$ is close to the identity $1_{\mathcal{S} X}$.

Finally, let $B \subseteq \mathcal{S} X$ be bounded. Then, as we remarked earlier, $B \subseteq X \times[0, a]$ for some $a \in[0,1)$. It follows that we have $N \in \mathbb{N}$ such that $t^{n}[\mathcal{S} X] \cap B=\varnothing$ for all $n \geq N$.

In conclusion, we see that the space $\mathcal{S} X$ is flasque.

Proposition 5.2 Let $X$ be a flasque coarse space. Then the closed cone $\mathcal{C} X$ is also flasque. 
Proof Let $t: X \rightarrow X$ be a coarse map with the required properties to make the space $X$ flasque. Let $\pi: X \times[0,1] \rightarrow \mathcal{C} X$ be the quotient map.

Define a map $\tilde{t}: \mathcal{C} X \rightarrow \mathcal{C} X$ by the formula

$$
\tilde{t}([x, s])=[t(x), s] .
$$

Let $\widetilde{M} \subseteq \mathcal{C} X \times \mathcal{C} X$ be a controlled set. Then $\widetilde{M} \subseteq \pi[M \times[0,1] \times[0,1]]$ where $M$ is a controlled set for the coarse space $X$; here we are indulging in some mild abuse of notation involving the order of factors. We know that the set $\bigcup_{n \in \mathbb{N}} t^{n}[M]$ is controlled. It follows that the set $\bigcup_{n \in \mathbb{N}} \widetilde{t}^{n}[\widetilde{M}]$ is controlled. In particular, we have shown that the map $\tilde{t}$ is controlled.

Now, the map $t$ is close to the identity map on the space $X$. Therefore the set

$$
M=\{(x, t(x)) \mid x \in X\}
$$

is controlled. Let

$$
\widetilde{M}=\{([x, s], \widetilde{t}[x, s]) \mid[x, s] \in \mathcal{C} X\} .
$$

Observe

$$
\widetilde{M}=\{([x, s],[t(x), s]) \mid[x, s] \in \mathcal{C} X\} \subseteq \pi[M \times[0,1] \times[0,1]]
$$

so the set $\widetilde{M}$ is also controlled, and the map $\tilde{t}$ is close to the identity map.

Finally, let $\widetilde{B} \subseteq \mathcal{C} X$ be bounded. As above, we have $\widetilde{B} \subseteq \pi[B \times[0,1]]$ for some bounded set $B \subseteq X$. Now we have a natural number $N$ such that $t^{n}[X] \cap B=\varnothing$ whenever $n \geq N$. Hence $\widetilde{t}^{n}[\mathcal{C} X] \cap \widetilde{B}=\varnothing$ whenever $n \geq N$.

Thus the space $\mathcal{C} X$ is also flasque, as claimed.

Lemma 5.3 Let $X$ be a Hausdorff space equipped with a coarse structure compatible with the topology. Let $E$ be a coarsely excisive functor. Then we have a weak fibration

$$
E(X) \stackrel{j}{\rightarrow} E(\mathcal{C} X) \stackrel{v}{\rightarrow} E(\mathcal{O} X) .
$$

Proof We have a coarsely excisive decomposition

$$
\mathcal{O} X=\frac{X \times[0,1 / 2]}{X \times\{0\}} \cup X \times\left[\frac{1}{2}, 1\right) .
$$

Observe that the first space in the decomposition is coarsely equivalent to the space $\mathcal{C} X$, and the second space is coarsely equivalent to the space $\mathcal{S} X$. By Proposition 5.1, the space $\mathcal{S} X$ is flasque, so the space $E(\mathcal{S} X)$ is weakly contractible. 
Hence, by the homotopy push-out property of the functor $E$, we have a weak fibration

$$
E(X) \rightarrow E(\mathcal{C} X) \rightarrow E(\mathcal{O} X)
$$

as claimed.

Definition 5.4 We call the boundary map

$$
\text { ว: } \Omega E(\mathcal{O} X) \rightarrow E(X)
$$

associated to the above weak fibration the coarse assembly map associated to the functor $E$.

Definition 5.5 Let $X$ be a coarse space. We say $X$ is uniformly locally finite if for any controlled set $M$, there is a number $k$ such that $|M(x)| \leq k$ for all $x \in X$.

We say $X$ has bounded geometry if it is coarsely equivalent to a uniformly locally finite coarse space.

For example, any subset of Euclidean space, $\mathbb{R}^{n}$, has bounded geometry.

Definition 5.6 Let $X$ be a coarse topological space. We call $X$ uniformly contractible if for every open controlled set $M$, there is a controlled set $N \supseteq M$ such that for all $x \in X$, the inclusion $M(x) \hookrightarrow N(x)$ is homotopic to the constant map onto the point $x$.

For example (see [36]), a contractible metric space on which some group acts cocompactly by isometries is uniformly contractible.

Definition 5.7 The coarse isomorphism conjecture associated to the functor $E$ asserts that the coarse assembly map is a stable equivalence whenever $X$ is a uniformly contractible space with bounded geometry.

Proposition 5.8 Let $E$ be a coarsely excisive functor. The coarse isomorphism conjecture holds for a space $X$ if and only if the spectrum $E(\mathcal{C} X)$ is weakly contractible.

Proof We have a long exact sequence

$$
\pi_{n+1} E(\mathcal{C} X) \stackrel{v_{*}}{\rightarrow} \pi_{n+1} E(\mathcal{O} X) \stackrel{\partial_{*}}{\rightarrow} \pi_{n} E(X) \stackrel{j_{*}}{\rightarrow} \pi_{n} E(\mathcal{C} X) .
$$

If the spectrum $E(\mathcal{C} X)$ is weakly contractible, then the groups $\pi_{n+1} E(\mathcal{C} X)$ and $\pi_{n} E(\mathcal{C} X)$ are both zero, so the map $\partial_{*}$ is an isomorphism. It follows that the coarse isomorphism conjecture holds for $X$. 
Conversely, suppose the coarse isomorphism conjecture holds for $X$. Then the above map $\partial_{*}$ is an isomorphism, and the maps $j_{*}$ and $v_{*}$ are both zero.

But we can extend the above long exact sequence to the right:

$$
\pi_{n} E(X) \stackrel{j_{*}}{\rightarrow} \pi_{n}(E(\mathcal{C} X)) \stackrel{v_{*}}{\rightarrow} \pi_{n} E(\mathcal{O} X)
$$

We know that $j_{*}=0, v_{*}=0$, and $\operatorname{im} j_{*}=\operatorname{ker} v_{*}$. Thus $\pi_{n} E(\mathcal{C} X)=0$, and the spectrum $E(\mathcal{C} X)$ is weakly contractible, as desired.

In $[27 ; 30]$, the author introduced the notion of a finite coarse CW-complex. The definition of a coarse CW-complex is similar to that of a CW-complex in topology, but the $n$-dimensional cells are products $(R \vee R)^{n} \times R$; the boundary of an $n$-cell is the space $(R \vee R)^{n} \times\{0\}$.

The following is an immediate consequence of the results of [27].

Theorem 5.9 Let $E$ be a coarsely excisive functor. Then the coarse isomorphism conjecture associated to $E$ holds for any finite coarse $C W$-complex.

As mentioned in the introduction, it is likely to be possible to extend this result.

\section{Examples}

Let $R$ be a ring. We call a category, $\mathcal{A}$, an $R$-algebroid (see [25]) if each morphism set $\operatorname{Hom}(a, b)_{\mathcal{A}}$ is a left $R$-module, and composition of morphisms

$$
\operatorname{Hom}(b, c)_{\mathcal{A}} \times \operatorname{Hom}(a, b)_{\mathcal{A}} \rightarrow \operatorname{Hom}(a, c)_{\mathcal{A}}
$$

is $R$-bilinear.

Let $\mathcal{A}$ be an $R$-algebroid, and consider objects $a, b \in \mathrm{Ob}(\mathcal{A})$. Then an object $a \oplus b$ is called a biproduct of the objects $a$ and $b$ if it comes equipped with morphisms $i_{a}: a \rightarrow a \oplus b, i_{b}: b \rightarrow a \oplus b, p_{a}: a \oplus b \rightarrow a$, and $p_{b}: a \oplus b \rightarrow b$ satisfying the equations

$$
p_{a} i_{a}=1_{a} \quad p_{b} i_{b}=1_{b} \quad i_{a} p_{a}+i_{b} p_{b}=1_{a \oplus b} .
$$

Observe that a biproduct is simultaneously a product and a coproduct. We call an $R$-algebroid $\mathcal{A}$ additive if it has a zero object (that is, an object that is simultaneously initial and terminal), and every pair of objects has a biproduct.

The following construction can be found in several articles on controlled algebraic $K-$ theory; see for example Anderson et al [1], Bartels [2] and Carlsson and Pedersen [6]. Our functorial approach comes from Weiss [38]. 
Definition 6.1 Let $X$ be a coarse space, and let $\mathcal{A}$ be an additive $R$-algebroid. Then a geometric $\mathcal{A}$-module over $X$ is a functor, $M$, from the collection of bounded subsets of $X^{2}$ to the category $\mathcal{A}$ such that for any bounded set $B$ the natural map

$$
\bigoplus_{x \in B} M(\{x\}) \rightarrow M(B)
$$

induced by the various inclusions is an isomorphism, and the support

$$
\operatorname{Supp}(M)=\{x \in X \mid M(\{x\}) \neq 0\}
$$

is a locally finite subset of $X$.

Here, by a subset $S \subseteq X$ being locally finite, we mean that $S \cap B$ is finite whenever $B$ is a bounded set.

For convenience, we write $M_{x}=M(\{x\})$. A morphism $\phi: M \rightarrow N$ between geometric $\mathcal{A}$-modules over $X$ is a collection of morphisms $\phi_{x, y}: M_{y} \rightarrow N_{x}$ in the category $\mathcal{A}$ such that for each fixed point $x \in X$, the morphism $\phi_{x, y}$ is nonzero for only finitely many points $y \in X$, and for each fixed point $y \in X$, the morphism $\phi_{x, y}$ is nonzero for only finitely many points $x \in X$.

Composition of morphisms $\phi: M \rightarrow N$ and $\psi: N \rightarrow P$ is defined by the formula

$$
(\psi \circ \phi)_{x, y}(\eta)=\sum_{z \in X} \psi_{x, z} \circ \phi_{z, y}(\eta)
$$

The local finiteness condition ensures this makes sense. We define the support of a morphism $\phi$

$$
\operatorname{Supp}(\phi)=\left\{(x, y) \in X \mid \phi_{x, y} \neq 0\right\}
$$

Definition 6.2 The category $\mathcal{A}[X]$ consists of all geometric $\mathcal{A}$-modules over $X$ and morphisms such that the support is controlled with respect to the coarse structure of $X$.

Observe that $\mathcal{A}[X]$ is again an additive $R$-algebroid. In particular, we can form its algebraic $K$-theory spectrum $\mathbb{K} \mathcal{A}(X)$ (constructed for instance by Pedersen and Weibel [33]). The algebraic $K$-theory groups $K_{n} \mathcal{A}(X)$ are the stable homotopy groups of this spectrum.

Given a coarse map $f: X \rightarrow Y$, we have an induced additive functor $f_{*}: \mathcal{A}[X] \rightarrow \mathcal{A}[Y]$. For a geometric $\mathcal{A}$-module, $M$, over $X$, we define a geometric $\mathcal{A}$-module $f_{*}[M]$ by writing $f_{*}[M](S)=M\left(f^{-1}[S]\right)$.

\footnotetext{
${ }^{2}$ Regarded as a category by looking at the usual partial ordering.
} 
Let $\phi: M \rightarrow N$ be a morphism in the category $\mathcal{A}[X]$. We define a morphism $f_{*}[\phi]: f_{*}[M] \rightarrow f_{*}[N]$ by writing

$$
f_{*}[\phi]_{h_{1}, h_{2}}=\sum_{\substack{g_{1} \in f^{-1}\left(h_{1}\right) \\ g_{2} \in f^{-1}\left(h_{2}\right)}} \phi_{g_{1}, g_{2}} .
$$

With these induced maps, we have a functor $X \mapsto \mathcal{A}[X]$, and so a functor $X \mapsto \mathbb{K} \mathcal{A}[X]$.

Theorem 6.3 The functor $X \mapsto \mathbb{K} \mathcal{A}[X]$ is coarsely excisive.

Proof To begin with, let $X$ be a coarse space, and let $p: X \rightarrow R$ be a coarse map to a generalised ray. Consider the inclusion $i_{0}: X \rightarrow I_{p} X$ defined by the formula $i(x)=(x, 0)$. Let $q: I_{p} X \rightarrow X$ be defined by the formula $q(x, t)=x$. Then certainly $q \circ i_{0}=1_{X}$, and $i_{0} \circ q(x, t)=(x, 0)$.

Let $M$ be a geometric $\mathcal{A}$-module over $I_{p} X$. Then

$$
\left(i_{0} \circ q\right)_{*}(E)_{(x, t)}= \begin{cases}0 & t \neq 0, \\ \bigoplus_{0 \leq t \leq p(x)+1} E_{(x, t)} & t=0 .\end{cases}
$$

It follows that we have a natural isomorphism $M \rightarrow\left(i_{0} \circ q\right)_{*}(M)$. Hence the functor $\left(i_{0}\right)_{*}: \mathcal{A}[X] \rightarrow \mathcal{A}\left[I_{p} X\right]$ is an equivalence of categories. The same argument proves that the functor $\left(i_{1}\right)_{*}: \mathcal{A}[X] \rightarrow \mathcal{A}\left[I_{p} X\right]$ where $i_{1}(x)=(x, p(x)+1)$ is also an equivalence of categories.

Certainly an equivalence of categories induces a homotopy equivalence at the level of $K$-theory spectra. We conclude that the functor $X \mapsto \mathbb{K} \mathcal{A}[X]$ takes coarse homotopy equivalences to homotopy equivalences. In particular, if two maps $f, g: X \rightarrow Y$ are coarsely equivalent, the maps $f_{*}, g_{*}: \mathbb{K} \mathcal{A}[X] \rightarrow \mathbb{K} \mathcal{A}[Y]$ are coarsely homotopic.

Now, let $X$ be flasque. Then we have a map $\tau: X \rightarrow X$ which gives us the flasqueness property. Given a geometric $\mathcal{A}$-module, $M$, the direct sum

$$
\bigoplus_{n=0}^{\infty} \tau_{*}^{n}[M]
$$

is also a geometric $\mathcal{A}$-module since the powers of $\tau$ eventually leave any bounded subset of $X$.

Given a morphism $f: M \rightarrow N$ in the category $\mathcal{A}[X]$, the fact that the union $\bigcup_{n=0}^{\infty} \tau^{n}[M]$ is controlled whenever $M \subseteq X \times X$ is a controlled subset tells us that we have an induced morphism

in the category $\mathcal{A}[X]$.

$$
\sum_{n=0}^{\infty} f_{*} \tau_{*}^{n}: \bigoplus_{n=0}^{\infty} \tau_{*}^{n}[M] \rightarrow \bigoplus_{n=0}^{\infty} \tau_{*}^{n}[N]
$$


Hence, by definition of the $K$-theory of an additive category (see for example Waldhausen [37]), given a $K$-theory class $\lambda \in K_{n} \mathcal{A}[X]$, we have an induced $K$-theory class

$$
\sum_{n=0}^{\infty} \tau_{*}^{n}[\lambda]=\lambda+\tau_{*}[\lambda]+\tau_{*}^{2}[\lambda]+\cdots \quad \in \mathbb{K} \mathcal{A}[X] .
$$

Now the map $\tau$ is close to the identity map on $X$. Hence, by the first part of the current proof, the map $\tau_{*}$ is the identity at the level of $K$-theory groups. We see that

$$
\sum_{n=0}^{\infty} \tau_{*}^{n}[\lambda]=\lambda+\lambda+\cdots=\lambda+(\lambda+\lambda+\cdots) .
$$

Thus, by an Eilenberg swindle, we see that $\lambda=0$. Thus $K_{n} \mathcal{A}[X]=0$, meaning the stable homotopy groups of the spectrum $\mathbb{K} \mathcal{A}[X]$ are all zero. Therefore the spectrum $\mathbb{K} \mathcal{A}[X]$ is weakly contractible.

We now look at homotopy push-out squares. Let $X=U \cup V$ be a coarsely excisive decomposition of a coarse space $X$. Consider the sequence

$$
0 \rightarrow \mathcal{A}[U \cap V] \stackrel{\left(i_{*}, j_{*}\right)}{\longrightarrow} \mathcal{A}[U] \oplus \mathcal{A}[V] \stackrel{k_{*}-l_{*}}{\longrightarrow} \mathcal{A}[X] \rightarrow 0
$$

where $i, j, k, l$ are the relevant inclusions.

Clearly the functor $\left(i_{*}, j_{*}\right)$ is faithful, the functor $k_{*}-l_{*}$ is surjective on each morphism set, and $\left(k_{*}-l_{*}\right) \circ\left(i_{*}, j_{*}\right)=0$.

We claim that the above sequence is a short exact sequence. Consider morphisms $\phi, \psi: M \rightarrow N$ between geometric $\mathcal{A}$-modules over $U$ and $V$ respectively such that $k_{*} \phi-l_{*} \psi=0$, and the supports of $\phi$ and $\psi$ are controlled.

We see that $\phi_{x, y}=0$ and $\psi_{x, y}=0$ unless $x, y \in U \cap V$, and $\phi_{x, y}=\psi_{x, y}$ when $x, y \in U \cap V$. Define a morphism $\theta:\left.\left.M\right|_{U \cap V} \rightarrow N\right|_{U \cap V}$ by restricting the morphism $\phi$. Then certainly $(\phi, \psi)=\left(i_{*}, j_{*}\right)(\theta)$.

The support of the morphism $\theta$ is certainly controlled. By the fibration theorem in algebraic $K$-theory (see [24; 37]). we have a fibration

$$
\mathbb{K} \mathcal{A}[U \cap V] \rightarrow \mathbb{K} \mathcal{A}[U] \vee \mathbb{K} \mathcal{A}[V] \rightarrow \mathbb{K} \mathcal{A}[X] .
$$

It follows that we have a homotopy push-out

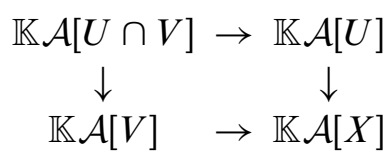

and we are done. 
The above result, along with Theorem 4.9, gives us a new proof of the fact that the functor $X \mapsto \mathbb{K} \mathcal{A}[\mathcal{O} X]$ is excisive. $^{3}$

Corollary 6.4 The set of functors $\left\{X \mapsto K_{n} \mathcal{A}[X] \mid n \in \mathbb{Z}\right\}$ on the category of coarse spaces is a coarse homology theory.

As observed in $[13 ; 27]$, one can construct a coarse homology theory by taking the analytic $K$-theory of the Roe $C^{*}$-algebra of a coarse space. However, this particular construction needs to be modified to make something that is functorial at the level of spectra.

Let $\mathcal{A}$ be a $\mathbb{C}$-algebroid. Then we call $\mathcal{A}$ a Banach category if each morphism set $\operatorname{Hom}(a, b)_{\mathcal{A}}$ is a Banach space, and given morphisms $x \in \operatorname{Hom}(a, b)_{\mathcal{A}}$ and $y \in$ $\operatorname{Hom}(b, c)_{\mathcal{A}}$, we have the inequality $\|y x\| \leq\|y\| \cdot\|x\|$.

An involution on a Banach category $\mathcal{A}$ is a collection of conjugate-linear maps $\operatorname{Hom}(a, b)_{\mathcal{A}} \rightarrow \operatorname{Hom}(b, a)_{\mathcal{A}}$, written $x \mapsto x^{*}$, such that $\left(x^{*}\right)^{*}=x$ for every morphism $x$, and $(x y)^{*}=y^{*} x^{*}$ whenever $x$ and $y$ are composable morphisms.

A Banach category with involution is termed a $C^{*}$-category if for every morphism $x \in \operatorname{Hom}(a, b)_{\mathcal{A}}$ the $C^{*}$-identity $\left\|x^{*} x\right\|=\|x\|^{2}$ holds, and the element $x^{*} x$ is a positive element of the $C^{*}$-algebra $\operatorname{Hom}(a, a)_{\mathcal{A}}$.

We refer the reader to Ghez, Lima and Roberts [10] and Mitchener [29] for more information on the theory of $C^{*}$-categories. As in Joachim [17] and Mitchener [28], one can associate a $K$-theory spectrum $\mathbb{K}(\mathcal{A})$ to a $C^{*}$-category $\mathcal{A}$. The stable homotopy groups of the $K$-theory spectrum of a $C^{*}$-algebra are the usual analytic $K$-theory groups of a $C^{*}$-algebra.

A functor between $C^{*}$-categories that is linear on each morphism set and compatible with the involution is called a $C^{*}$-functor. A $C^{*}$-functor $\alpha: \mathcal{A} \rightarrow \mathcal{B}$ is automatically continuous on each morphism set, with norm at most one, and induces a map $\alpha_{*}: \mathbb{K}(\mathcal{A}) \rightarrow \mathbb{K}(\mathcal{B})$ of $K$-theory spectra. With these induced maps, formation of the $K$-theory spectrum defines a functor, $\mathbb{K}$, from the category of $C^{*}$-categories and $C^{*}$-functors to the category of spectra.

The functor $\mathbb{K}$ takes short exact sequences of $C^{*}$-categories to fibrations of spectra, and takes equivalences of $C^{*}$-categories to homotopy equivalences of spectra.

The following definition is a slight generalisation of a construction inspired by [12, Section 2.2].

\footnotetext{
${ }^{3}$ For other proofs of this fact, see for instance Anderson et al [1] and Weiss [38].
} 
Definition 6.5 Let $\mathcal{A}$ be an additive $C^{*}$-category. Then we define $\mathcal{A}^{b}[X]$ to be the category of geometric $\mathcal{A}$-modules over $X$, and morphisms $\phi: M \rightarrow N$ such that the linear map

$$
T_{\phi}: \bigoplus_{x \in X} M_{x} \rightarrow \bigoplus_{x \in X} N_{x}
$$

defined by the formula

$$
T_{\phi}(v)=\sum_{y \in X} \phi_{x, y}(v), \quad v \in M_{x}
$$

is bounded. In this case, we define the norm of the morphism $\phi$ by writing $\|\phi\|=\left\|T_{\phi}\right\|$.

Observe that $\mathcal{A}^{b}[X]$ is a pre- $C^{*}$-category in the sense that it has all of the properties required of a $C^{*}$-category apart from the morphism sets being complete. As explained in [29], we can therefore complete it to form a $C^{*}$-category, which we label $\mathcal{A}^{*}[X]$.

The following result can now be proved in the same way as Theorem 6.3.

Theorem 6.6 Let $\mathcal{A}$ be a $C^{*}$-category. Then the functor $X \mapsto \mathbb{K} \mathcal{A}^{*}[X]$ is coarsely excisive.

The classical coarse Baum-Connes conjecture, as described for instance by Higson and Roe [14], can be considered the prototype of the other conjectures in this article. In that conjecture, the right-hand side is the $K$-theory of the Roe $C^{*}$-algebra, $C^{*}(X)$, of a coarse space $X$.

The following result is proved in the discussion in Section 2.2 of [12].

Theorem 6.7 Let $\mathcal{V}$ be the $C^{*}$-category where the objects are the Hilbert spaces $\mathbb{C}^{n}$, and the morphisms are bounded linear maps. Let $X$ be a separable coarse topological space with bounded geometry.

Then we have isomorphisms $\pi_{n} \mathbb{K} \mathcal{V}^{*}[X] \cong K_{n} C^{*}(X)$.

Actually, the spectrum on the left is built using for instance a topological version of Waldhausen's $S_{\bullet}-$ construction from [37]. This agrees with the analytic approach to $K$-theory spectra in [28]; see [26] for details.

The above establishes that we have a functor $X \mapsto \mathbb{K}^{*}[X]$ where the stable homotopy groups are isomorphic to the $K$-theory groups of the Roe $C^{*}$-algebra. Note that it is far from obvious if looked at directly to see that the assignment $X \mapsto \mathbb{K} C^{*}(X)$ is a functor. 
In any case, we have an assembly map

$$
\Omega \mathbb{K} \mathcal{V}^{*}[\mathcal{O} X] \rightarrow \mathbb{K} \mathcal{V}^{*}[X]
$$

where the domain and codomain of this map agree with the domain and codomain of the map in the coarse Baum-Connes conjecture [14]. However, we do not yet know whether the map itself agrees with the coarse Baum-Connes conjecture, although similar methods of proof apply, and the map being an isomorphism has similar consequences. We show in Theorem 8.8 that an equivariant version of this map is the same, up to stable equivalence, as the usual Baum-Connes assembly map.

\section{Equivariant assembly}

Let $G$ be a discrete group.

Definition 7.1 A coarse space equipped with an action of the group $G$ by coarse maps is termed a coarse $G$-space.

We will assume that a group $G$ acts on the right of a space. We call a subset, $A$, of a coarse $G$-space $X$ cobounded if there is a bounded subset $B \subseteq X$ such that $A \subseteq B \cdot G$.

Definition 7.2 The coarse $G$-category is the category where the objects are coarse $G$-spaces, and the morphisms are controlled equivariant maps where the inverse image of a cobounded set is cobounded.

If $X$ is a coarse $G$-space, and $p: X \rightarrow R$ is a map to a generalised ray, the group $G$ acts on the cylinder $I_{p} X$ by writing $(x, t) g=(x g, t)$. The inclusions $i_{0}, i_{1}: X \rightarrow I_{p} X$ are morphisms in the coarse $G$-category.

Definition 7.3 Let $f_{0}, f_{1}: X \rightarrow Y$ be morphisms between coarse $G$-spaces. An elementary coarse $G$-homotopy between $f_{0}$ and $f_{1}$ is an equivariant coarse map $H: I_{p} X \rightarrow Y$ for some $p: X \rightarrow R$ such that $f_{0}=H \circ i_{0}$ and $f_{1}=H \circ i_{1}$.

More generally, we call the maps $f_{0}$ and $f_{1}$ coarsely $G$-homotopic if they can be linked by a chain of elementary coarse homotopies.

A morphism $f: X \rightarrow Y$ in the coarse $G$-category is termed a coarse $G$-homotopy equivalence if there is a morphism $g: Y \rightarrow X$ such that the compositions $g \circ f$ and $f \circ g$ are coarsely $G$-homotopic to the identities $1_{X}$ and $1_{Y}$ respectively. 
Definition 7.4 We call a coarse $G$-space $X \quad G$-flasque if there is an equivariant map $t: X \rightarrow X$ such that:

- Let $B \subseteq X$ be bounded. Then there exists $N \in \mathbb{N}$ such that $t^{n}[X] \cap B=\varnothing$ for all $n \geq N$.

- Let $M \subseteq X \times X$ be controlled. Then the union $\bigcup_{n \in \mathbb{N}} t^{n}[M]$ is controlled.

- The map $t$ is close to the identity map.

Note that the above map $t$ is controlled by the above axioms.

As in the nonequivariant case, if a coarse $G$-space $X$ is $G$-flasque, then so is the closed cone $\mathcal{C} X$. If $X$ is any coarse $G$-space, the open square $\mathcal{S} X$ is $G$-flasque.

Definition 7.5 We call a functor $E_{G}$ from the coarse $G$-category to the category of spectra coarsely $G$-excisive if the following conditions hold.

- The spectrum $E_{G}(X)$ is weakly contractible whenever the coarse space $X$ is $G$-flasque.

- The functor $E_{G}$ takes coarse $G$-homotopy equivalences to weak homotopy equivalences of spectra.

- Given a coarsely excisive decomposition $X=A \cup B$, where $A$ and $B$ are coarse $G$-spaces, we have a homotopy push-out diagram

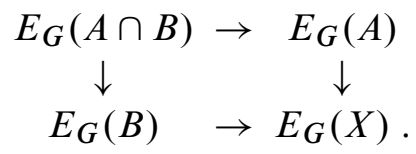

- Let $X$ be a cobounded coarse $G$-space. Then the constant map $c: X \rightarrow+$ induces a stable equivalence $c_{*}: E_{G}(X) \rightarrow E_{G}(+)$.

Our next definition comes from Davis and Lück [7].

Definition 7.6 Let $\mathcal{C}_{G}$ be the category of topological spaces equipped with a $G$-action that are homotopy equivalent to $G-\mathrm{CW}$-complexes. We call a functor, $F$, from the category $\mathcal{C}_{G}$ to the category of spectra $G$-excisive if it takes $G$-homotopy equivalences of spaces to homotopy equivalences of spectra, and given a space $X=U \cup V$, where $U$ and $V$ are $G$-invariant open sets we have a homotopy push-out diagram

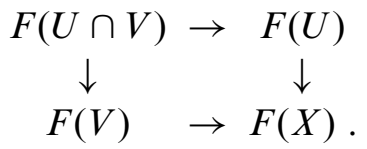


Recall that we call a $G$-space $X$ cocompact if there is a compact subset $K \subseteq X$ such that $X=K G$. The following is proved similarly to Theorem 4.9:

Theorem 7.7 Let $E_{G}$ be a coarsely $G$-excisive functor. Then the assignment $X \mapsto$ $E_{G}(\mathcal{O} X)$ defines a $G$-excisive functor on the category of cocompact Hausdorff $G$ spaces.

The following is immediate.

Proposition 7.8 Let $E_{G}$ be a coarsely $G$-excisive functor. Then the sequence of functors $X \mapsto \pi_{n} E_{G}(\mathcal{O} X)$ forms a $G$-homology theory.

By a $G$-homology theory, we mean one that satisfies equivariant analogues of the Eilenberg-Steenrod axioms; see Kreck and Lück [20] for a discussion. In fact, we could go further and show that the collection of functors $X \mapsto \pi_{n} E_{G}(\mathcal{O} X)$ for different groups $G$ defines an equivariant homology theory, but we do not need this stronger result here.

The following is similar to Lemma 5.3.

Lemma 7.9 Let $X$ be a coarse Hausdorff $G$-space. Let $E_{G}$ be a coarsely $G$-excisive functor. Then we have a weak fibration

$$
E_{G}(X) \stackrel{j}{\longrightarrow} E_{G}(\mathcal{C} X) \stackrel{v_{G}}{\longrightarrow} E_{G}(\mathcal{O} X) .
$$

So we have an associated boundary map

$$
\partial_{G}: \Omega E_{G}(\mathcal{O} X) \rightarrow E_{G}(X) .
$$

We call this map the equivariant assembly map associated to the functor $E_{G}$.

Recall that a $G$-space, $X$, is termed free if for every point $x \in X$, we have $x g=x$ only when $g=e$, where $e$ denotes the identity element of a group $G$. We define $E G$ to be a weakly contractible free $G-\mathrm{CW}$-complex. The space $E G$ is unique up to $G$-homotopy equivalence, and the quotient space $E G / G$ is the classifying space $B G$.

Definition 7.10 The Novikov conjecture associated to the functor $E_{G}$ asserts that the equivariant assembly map is injective at the level of stable homotopy groups when $X=E G$ for some coarse structure on $E G$ compatible with the topology. 
More generally, let $\mathcal{F}$ be a family of subgroups of a group $G$ (that is a collection of subgroups closed under the operations of conjugation and finite intersections). For instance, we can consider all finite subgroups, or all virtually cyclic subgroups. We call a $G$-CW-complex $X$ a $(G, \mathcal{F})-C W$-complex if the fixed point set $X^{H}$ is empty if $H \notin \mathcal{F}$.

Each cell in a $(G, \mathcal{F})-\mathrm{CW}$-complex is a $G$-space, $C$, with the property that $C^{H}=\varnothing$ if $H \notin \mathcal{F}$.

We can form a unique (up to $G$-homotopy) $(G, \mathcal{F})$-CW-complex $E(G, \mathcal{F})$, with the property that the fixed point set $E(G, \mathcal{F})^{H}$ is $G$-contractible if $H \in \mathcal{F}$.

As a special case, if the family $\mathcal{F}$ consists of just the trivial subgroup of $G$, then $E(G, \mathcal{F})=E G$. If the family $\mathcal{F}$ consists of all finite subgroups, then $E(G, \mathcal{F})$ is the classifying space $\underline{E} G$ described for instance by Baum, Connes and Higson [4].

It is shown in [7] that the space $E(G, \mathcal{F})$ is a classifying space for $(G, \mathcal{F})-\mathrm{CW}$ complexes in the following sense.

Theorem 7.11 Let $\mathcal{F}$ be a family of subgroups of a group $G$. Let $X$ be a $(G, \mathcal{F})-$ $C W$-complex. Then we have an equivariant map $u: X \rightarrow E(G, \mathcal{F})$, and any two such maps are $G$-homotopic.

Definition 7.12 The $\left(E_{G}, \mathcal{F}\right)$-isomorphism conjecture asserts that the equivariant assembly map $\partial_{G}: \Omega E_{G}(\mathcal{O} X) \rightarrow E_{G}(X)$ is a stable equivalence when $X=E(G, \mathcal{F})$ for some coarse structure on $E(G, \mathcal{F})$ compatible with the topology.

We will identify examples of these conjectures in the next section.

Definition 7.13 Let $E$ be a coarsely excisive functor. Then we say a coarsely $G-$ excisive functor $E_{G}$ has the local property relative to $E$ if there is a natural map $i: E_{G}(X) \rightarrow E(X)$, such that if $X=\mathcal{O} Y$, where $Y$ is a free coarse cocompact $G$-space, and $\pi: X \rightarrow X / G$ is the quotient map, then the composite

$$
\pi_{*} \circ i=i \circ \pi_{*}: E_{G}(X) \rightarrow E(X / G)
$$

is a stable equivalence.

In particular, if the functor $E_{G}$ has the local property relative to $E$, then the assembly map in the Novikov conjecture can be regarded as a map

$$
\partial_{G}: \Omega E(\mathcal{O} B G) \rightarrow E_{G}(E G) .
$$


Proposition 7.14 Let $E_{G}$ be a coarsely $G$-excisive functor with the local property relative to $E$. Suppose that the assembly map in the $\left(E_{G}, \mathcal{F}\right)$-isomorphism conjecture is injective at the level of stable homotopy groups for some family $\mathcal{F}$, and the spaces $E G$ and $E(G, \mathcal{F})$ are both cocompact. Then the Novikov conjecture holds for the functor $E_{G}$.

Proof Certainly, the space $E G$ is a $(G, \mathcal{F})-\mathrm{CW}$-complex, therefore we have a map $u: E G \rightarrow E(G, \mathcal{F})$. On the other hand, the space $E(G, \mathcal{F})$ is a $G$-space, so we have a map $v: E(G, \mathcal{F}) \rightarrow B G$. Since $E G$ is a $G$-space, up to $G$-homotopy there is only one equivariant map $E G \rightarrow B G$. But $B G=E G / G$, so both $v \circ u$ and the quotient map $\pi$ are such maps.

We conclude that the quotient map $\pi$ and the composite $v \circ u$ are $G$-homotopic. Since the functor $E_{G}$ has the local property, and $E G$ is a free $G$-space, we see that the composite $v_{*} \circ u_{*}: E_{G}(\mathcal{O} E G) \rightarrow E(\mathcal{O} B G)$ is a stable equivalence. In particular, the functor $u_{*}: E_{G}(\mathcal{O} E G) \rightarrow E_{G}(\mathcal{O} E(G, \mathcal{F}))$ is split injective at the level of stable homotopy groups.

Now, the map $u$ induces a commutative diagram

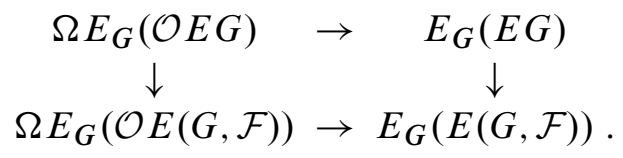

We saw above that the vertical arrow on the left is split injective at the level of stable homotopy groups, and by hypothesis, the map at the bottom is injective. So the map at the top is also injective at the level of stable homotopy groups. But this statement is the Novikov conjecture.

\section{Equivariant examples}

Let $X$ be a coarse $G$-space, let $R$ be a ring, and let $\mathcal{A}$ be an additive $R$-algebroid. Then we call a geometric $\mathcal{A}$-module, $M$, over $X \quad G$-invariant if $M_{x g}=M_{x}$ for all $x \in X$ and $g \in G$. A morphism $\phi: M \rightarrow N$ between such modules is termed $G$-invariant if $\phi_{x g, y g}=\phi_{x, y}$ for all $x, y \in X$.

Definition 8.1 We write $\mathcal{A}_{G}[X]$ to denote the category of $G$-invariant geometric $\mathcal{A}$-modules over $X$, and $G$-invariant morphisms.

The following result is similar to Theorem 6.3.

Theorem 8.2 The assignment $X \mapsto \mathbb{K} \mathcal{A}_{G}[X]$ is a coarsely $G$-excisive functor. 
The following is obvious from the definition.

Proposition 8.3 The functor $\mathbb{K} \mathcal{A}_{G}$ has the local property relative to the functor $\mathcal{A}$.

Of course, it follows from the above that the functor $X \mapsto \mathbb{K} \mathcal{A}_{G}[\mathcal{O} X]$ is $G$-excisive. An alternative proof of this can be found for instance in [3].

Definition 8.4 Let $X$ be a coarse $G$-space, and let $\mathcal{A}$ a $C^{*}$-category. Then we define $\mathcal{A}_{G}^{b}[X]$ to be the category of $G$-invariant geometric $\mathcal{A}$-modules over $X$, and $G$-invariant bounded morphisms.

We define $\mathcal{A}_{G}^{*}[X]$ to be the $C^{*}$-category we obtain by completion.

Similarly to Theorem 6.6 , we have the following:

Theorem 8.5 Let $\mathcal{A}$ be a $C^{*}$-category. Then the functor $X \mapsto \mathbb{K} \mathcal{A}_{G}^{*}[X]$ is coarsely $G$-excisive.

Again, the functor $\mathbb{K} \mathcal{A}_{G}^{*}$ has the local property relative to the functor $\mathbb{K} \mathcal{A}^{*}$.

Now (see for instance [15]), given a coarse $G$-space $X$ equipped with a topology compatible with the coarse structure, there is an equivariant analogue of the Roe $C^{*}$-algebra, $C_{G}^{*}(X)$.

Let $|G|$ be the coarse space associated to a finitely presented group $G$, by picking a word length metric. ${ }^{4}$ Then it is shown in [35] that the $C^{*}$-algebra $C_{G}^{*}|G|$ has the same $K$-theory as the reduced group $C^{*}$-algebra $C_{r}^{*} G$.

Just as in the nonequivariant case, we have the following.

Theorem 8.6 Let $X$ be a coarse $G$-space equipped with a topology compatible with the coarse structure. Suppose we have a separable coarse space, $Y$, with bounded geometry such that $X=Y \cdot G$.

Then we have isomorphisms $K_{n} \mathcal{V}_{G}^{*}[X] \cong K_{n} C_{G}^{*}(X)$.

Now, fix an algebroid $\mathcal{A}$, and consider the $G$-excisive functor defined by the formula

$$
F_{G}(X)=\Omega \mathbb{K} \mathcal{A}_{G}[\mathcal{O} X] .
$$

According to [7], the Farrell-Jones assembly map in algebraic $K$-theory is the map $c: F_{G}(X) \rightarrow F_{G}(+)$ induced by the constant map $X \rightarrow+$. The following result closely follows the methods described in [12] for controlled assembly.

\footnotetext{
${ }^{4}$ The particular choice of word length metric does not affect the coarse structure.
} 
Theorem 8.7 Let $G$ act cocompactly on the space $X$. Then the Farrell-Jones assembly map $c: F_{G}(X) \rightarrow F_{G}(+)$ is stably-equivalent to the equivariant assembly map $\partial_{G}: \Omega \mathbb{K} \mathcal{A}_{G}[\mathcal{O} X] \rightarrow \mathbb{K} \mathcal{A}_{G}[X]$.

Proof Consider the commutative diagram

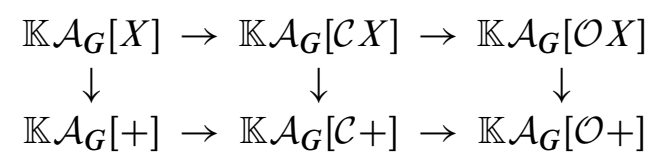

where the vertical maps are all induced by the constant map $X \rightarrow+$. Since the $G$-space $X$ is cocompact, it is cobounded as a coarse $G$-space, so the constant map $X \rightarrow+$ is a morphism in the coarse $G$-category.

The rows of the above diagram are fibrations. The space $\mathcal{C}+$ is clearly flasque, so the spectrum $\mathbb{K} \mathcal{A}_{G}[\mathcal{C}+]$ is weakly contractible. We therefore have a commutative diagram

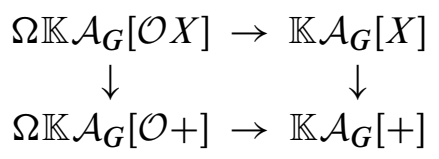

where the bottom row is a stable equivalence.

Since the group $G$ acts cocompactly on the space $X$, there is a bounded set $K$ such that $G K=X$, and $x g \notin K$ if $x \in K$ and $g \neq e$. Thus, if we give the space $K$ the trivial $G$-action, by definition of the functor $\mathcal{A}_{G}$, the categories $\mathcal{A}_{G}[X]$ and $\mathcal{A}_{G}[K]$ are equivalent.

But the spaces $K$ and + are equivariantly coarsely equivalent, and so equivariant coarse homotopy equivalent. Hence the map $a: \mathbb{K} \mathcal{A}_{G}[X] \rightarrow \mathbb{K} \mathcal{A}_{G}[+]$ is a stable equivalence.

The desired result now follows.

The corresponding result for the Baum-Connes assembly map follows similarly, although we need the fact that the assembly map fits into the picture in [7]; see for example [31].

Theorem 8.8 Let $G$ act cocompactly on the space $X$. Then the Baum-Connes assembly map for the $G$-space $X$ is stably-equivalent to the equivariant assembly map $\partial_{G}: \Omega \mathbb{K} \mathcal{V}_{G}^{*}[\mathcal{O} X] \rightarrow \mathbb{K} \mathcal{V}_{G}^{*}[X]$ 


\section{Descent}

Let $G$ be a discrete group, and let $A$ be a $G$-spectrum. Then we define the homotopy fixed point spectrum, $A^{h G}$, to be the spectrum of continuous equivariant maps $\mu: E G \rightarrow A$. Each space in the spectrum $A^{h G}$ has the compact open topology.

The following result is well-known.

Proposition 9.1 Let $A$ and $B$ be $G$-spectra. Let $f: A \rightarrow B$ be a (nonequivariant) weak homotopy equivalence. Then the obvious induced map $f_{*}: A^{h G} \rightarrow B^{h G}$ is also a weak homotopy equivalence.

In particular, if the spectrum $A$ is weakly contractible, then so is the spectrum $A^{h G}$.

A proof of the second assertion (at least for spaces) can be found in [34]; the first then follows by looking at mapping cones.

Applying the above to a coarsely excisive functor immediately gives us the following.

Proposition 9.2 Let $E$ be a coarsely excisive functor. Then the functor $X \mapsto E(X)^{h G}$, defined on the category of coarse $G$-spaces and equivariant coarse maps, is coarsely $G$-excisive.

Thus the sequence of functors $X \mapsto \pi_{n} E(\mathcal{O} X)^{h G}$ forms a $G$-homology theory, and we have a boundary map

$$
\partial_{h G}: \Omega E(\mathcal{O} X)^{h G} \rightarrow E(X)^{h G} .
$$

Lemma 9.3 Let $E_{G}$ be a coarsely $G$-excisive functor with the local property relative to a functor $E$. Then we have a natural transformation $j: E_{G}(X) \rightarrow E(X)^{h G}$.

Further, let $X=\bigvee_{g \in G} Y_{g}$, where each space $Y_{g}$ is a copy of the same coarse space $Y$, which is a cone of some compact space, and the group $G$ acts by permutations. Then the map $j: E_{G}(X) \rightarrow E(X)^{h G}$ is an isomorphism.

Proof By hypothesis, we have a natural map $i: E_{G}(X) \rightarrow E(X)$.

Observe we have a natural homotopy equivalence

$$
\begin{aligned}
E_{G}(X) & \simeq \operatorname{Map}\left(E G, E_{G}(X)\right) \\
& =\operatorname{Map}\left(E G, \operatorname{Map}_{G}\left(G, E_{G}(X)\right)\right) \\
& =\operatorname{Map}_{G}\left(E G, \operatorname{Map}\left(G, E_{G}(X)\right)\right) \\
& =\operatorname{Map}\left(G, E_{G}(X)\right)^{h G} .
\end{aligned}
$$


We have a natural map $\operatorname{Map}\left(G, E_{G}(X)\right) \rightarrow E(X)$ defined by composing the evaluation of a map $f: G \rightarrow E_{G}(X)$ at the identity element $1 \in G$, with the natural map $i$.

Taking homotopy fixed point sets, we have a natural transformation $j: E_{G}(X) \rightarrow$ $E(X)^{h G}$.

Now, let $X=\bigvee_{g \in G} Y_{g}$ as above. Let $Y=\mathcal{O} Z$. Then $X=\mathcal{O}(Z \times G)$.

Let $\pi: X \rightarrow Y$ be the quotient map. Then by the local property, the map $\pi_{*} \circ i=$ $i \circ \pi_{*}: E_{G}(X) \rightarrow E(Y)$ is a stable equivalence. Now, looking at disjoint unions,

$$
E(X)=\bigvee_{g \in G} E\left(Y_{g}\right)=\operatorname{Map}(G, E(Y)) .
$$

So in this case the above map $\operatorname{Map}\left(G, E_{G}(X)\right) \rightarrow E(X)=\operatorname{Map}(G, E(Y))$ is weakly homotopic to the stable equivalence $\operatorname{Map}\left(G, E_{G}(X)\right) \rightarrow \operatorname{Map}(G, E(Y))$ induced by the composite $\pi_{*} \circ i$. In particular, it follows that our map is a stable equivalence.

Taking homotopy fixed points, the map

$$
j: E_{G}(X) \rightarrow E(X)^{h G}
$$

is also a stable equivalence, and we are done.

So we have natural maps $i_{1}: E_{G}(\mathcal{C} X) \rightarrow E(\mathcal{C} X)^{h G}$ and $i_{2}: E_{G}(\mathcal{O} X) \rightarrow E(\mathcal{O} X)^{h G}$ fitting into a commutative diagram

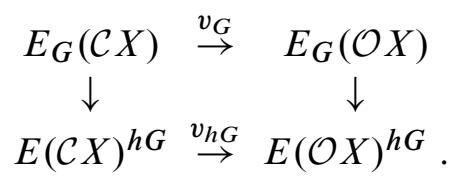

Proposition 9.4 The map $i_{2}: E_{G}(\mathcal{O} X) \rightarrow E(\mathcal{O} X)^{h G}$ is a stable equivalence whenever $X$ is a finite cocompact free $G-C W$-complex.

Proof Let $h_{*}^{G}$ be a $G$-homology theory. Let $X$ be a finite free $G-\mathrm{CW}$-complex. Then the equivariant version of the Atiyah-Hirzebruch spectral sequence (see for instance [23]) gives us a half-plane spectral sequence, $\left\{E_{p, q}^{*}\right\}$, that converges to $h_{*}^{G}(X)$, with $E^{2}$-term $E_{p, q}^{2} \cong H_{p}^{G}\left(X ; h_{q}^{G}\left(\Delta^{0}\right)\right)$, that is classical $G$-homology with coefficients in the group $h_{q}^{G}\left(\Delta_{G}^{0}\right)$, where $\Delta_{G}^{0}$ is the free 0 -dimensional $G$-cell.

Further, this spectral sequence depends functorially on the CW-complex $X$ and $G-$ homology theory $h_{*}^{G}$. The space $\Delta_{G}^{0}$ is just a copy of the group $G$, which acts on itself by right-translations.

The induced map $\left(i_{2}\right)_{*}: \pi_{*} E_{G}(\mathcal{O} X) \rightarrow E(\mathcal{O} X)^{h G}$ is a map between $G$-homology theories. By the above, it is an isomorphism when the space $X$ is a 0 -dimensional free 
$G$-cell. Thus the corresponding Atiyah-Hirzebruch spectral sequences are isomorphic, and the map $\left(i_{2}\right)_{*}$ is an isomorphism for any finite free $G-\mathrm{CW}-$ complex.

We now have the ingredients to prove our main result.

Theorem 9.5 Let $E_{G}$ be a coarsely $G$-excisive functor. Let $X$ be a free coarse $G$-space, that is, as a topological space, $G$-homotopy equivalent to a finite $G-C W$ complex.

Suppose the coarse isomorphism conjecture holds for the functor $E$ and the space $X$. Then the map $\partial_{G}: \Omega E_{G}(\mathcal{O} X) \rightarrow E_{G}(X)$ is injective at the level of stable homotopy groups.

Proof By Proposition 5.8, the coarse isomorphism conjecture for the space $X$ tells us that the spectrum $E(\mathcal{C} X)$ is weakly contractible.

Hence, by Proposition 9.1, the spectrum $E(\mathcal{C} X)^{h G}$ is also weakly contractible.

Now we have a commutative diagram

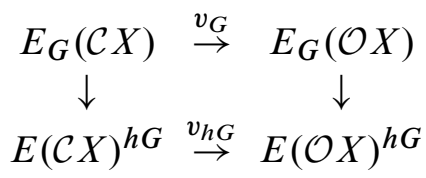

and by Proposition 9.4 the map $i_{2}: E_{G}(\mathcal{O} X) \rightarrow E(\mathcal{O} X)^{h G}$ is a weak equivalence.

Hence the map $v_{G}$ must be zero at the level of stable homotopy groups. The weak fibration in Lemma 7.9 gives us a long exact sequence

$$
\pi_{n+1} E_{G}(\mathcal{C} X) \stackrel{\left(v_{G}\right)_{*}}{\longrightarrow} \pi_{n+1} E_{G}(\mathcal{O} X) \stackrel{\left(\partial_{G}\right)_{*}}{\longrightarrow} \pi_{n} E_{G}(X) .
$$

As we have just mentioned, the map $\left(v_{G}\right)_{*}$ is zero. Thus the map $\left(\partial_{G}\right)_{*}$ is injective.

Applying the above result to the examples in the previous section, we immediately obtain the following.

Corollary 9.6 Let $X$ be a free coarse $G$-space, that is, as a topological space, $G$ homotopy equivalent to a finite $G-C W$-complex.

Let $R$ be a ring, and let $\mathcal{A}$ be an additive $R$-algebroid. Suppose the coarse assembly map $\partial: \Omega \mathbb{K} \mathcal{A}(\mathcal{O} X) \rightarrow \mathbb{K} \mathcal{A}(X)$ is an isomorphism. Then the Farrell-Jones assembly map is injective for the space $X, R$-algebroid $\mathcal{A}$ and group $G$. 
Corollary 9.7 Let $X$ be a free coarse $G$-space, that is, as a topological space, $G$ homotopy equivalent to a finite $G-C W$-complex.

Suppose the coarse assembly map $\partial: \Omega \mathbb{K} \mathcal{V}^{*}(\mathcal{O} X) \rightarrow \mathbb{K} \mathcal{V}^{*}(X)$ is an isomorphism. Then the Baum-Connes assembly map is injective for the space $X$ and group $G$.

\section{References}

[1] D R Anderson, F X Connolly, S C Ferry, E K Pedersen, Algebraic K-theory with continuous control at infinity, J. Pure Appl. Algebra 94 (1994) 25-47 MR1277522

[2] A C Bartels, Squeezing and higher algebraic K-theory, K-Theory 28 (2003) 19-37 MR1988817

[3] A C Bartels, F T Farrell, L E Jones, H Reich, On the isomorphism conjecture in algebraic K-theory, Topology 43 (2004) 157-213 MR2030590

[4] P Baum, A Connes, $\mathbf{N}$ Higson, Classifying space for proper actions and $K$-theory of group $C^{*}$-algebras, from: " $C^{*}$-algebras: 1943-1993 (San Antonio, TX, 1993)”, (R S Doran, editor), Contemp. Math. 167, Amer. Math. Soc. (1994) 240-291 MR1292018

[5] M Bökstedt, W C Hsiang, I Madsen, The cyclotomic trace and algebraic $K$-theory of spaces, Invent. Math. 111 (1993) 465-539 MR1202133

[6] G Carlsson, E K Pedersen, Controlled algebra and the Novikov conjectures for $\mathrm{K}$ and L-theory, Topology 34 (1995) 731-758 MR1341817

[7] J F Davis, W Lück, Spaces over a category and assembly maps in isomorphism conjectures in $K$ - and L-theory, $K$-Theory 15 (1998) 201-252 MR1659969

[8] A N Dranishnikov, Asymptotic topology, Uspekhi Mat. Nauk 55 (2000) 71-116 MR1840358

[9] F T Farrell, LE Jones, Isomorphism conjectures in algebraic $K$-theory, J. Amer. Math. Soc. 6 (1993) 249-297 MR1179537

[10] P Ghez, R Lima, J E Roberts, $W^{*}$-categories, Pacific J. Math. 120 (1985) 79-109 MR808930

[11] E Guentner, $\mathbf{R}$ Tessera, $\mathbf{G} \mathbf{Y u}$, A notion of geometric complexity and its application to topological rigidity arXiv:1008.0884v1

[12] I Hambleton, E K Pedersen, Identifying assembly maps in $K$ - and L-theory, Math. Ann. 328 (2004) 27-57 MR2030369

[13] N Higson, E K Pedersen, J Roe, $C^{*}$-algebras and controlled topology, $K$-Theory 11 (1997) 209-239 MR1451755

[14] N Higson, J Roe, On the coarse Baum-Connes conjecture, from: "Novikov conjectures, index theorems and rigidity, Vol. 2 (Oberwolfach, 1993)", (S C Ferry, A Ranicki, J Rosenberg, editors), London Math. Soc. Lecture Note Ser. 227, Cambridge Univ. Press (1995) 227-254 MR1388312 
[15] N Higson, J Roe, Analytic K-homology, Oxford Math. Monogr., Oxford Sci. Publ., Oxford Univ. Press, Oxford (2000) MR1817560

[16] N Higson, J Roe, G L Yu, A coarse Mayer-Vietoris principle, Math. Proc. Cambridge Philos. Soc. 114 (1993) 85-97 MR1219916

[17] M Joachim, $K$-homology of $C^{*}$-categories and symmetric spectra representing $K-$ homology, Math. Ann. 327 (2003) 641-670 MR2023312

[18] G G Kasparov, Equivariant $K K$-theory and the Novikov conjecture, Invent. Math. 91 (1988) 147-201 MR918241

[19] G G Kasparov, K-theory, group $C^{*}$-algebras, and higher signatures (conspectus), from: "Novikov conjectures, index theorems and rigidity, Vol. 1 (Oberwolfach, 1993)", (S C Ferry, A Ranicki, J Rosenberg, editors), London Math. Soc. Lecture Note Ser. 226, Cambridge Univ. Press (1995) 101-146 MR1388299

[20] M Kreck, W Lück, The Novikov conjecture. Geometry and algebra, Oberwolfach Seminars 33, Birkhäuser Verlag, Basel (2005) MR2117411

[21] J-L Loday, $K$-théorie algébrique et représentations de groupes, Ann. Sci. École Norm. Sup. (4) 9 (1976) 309-377 MR0447373

[22] V-T Luu, Coarse categories $i$ : Foundations arXiv:0708.3901

[23] T Matumoto, Equivariant cohomology theories on G-CW-complexes, Osaka J. Math. 10 (1973) 51-68 MR0343259

[24] R McCarthy, On fundamental theorems of algebraic K-theory, Topology 32 (1993) 325-328 MR1217072

[25] B Mitchell, Separable algebroids, Mem. Amer. Math. Soc. 57 (1985) iv+96 MR804210

[26] P D Mitchener, Algebraic $K$-theory spectra and factorisations of analytic assembly maps arXiv:0711.2182

[27] P D Mitchener, Coarse homology theories, Algebr. Geom. Topol. 1 (2001) 271-297 MR1834777

[28] P D Mitchener, Symmetric $K$-theory spectra of $C^{*}$-categories, $K$-Theory 24 (2001) 157-201 MR1869627

[29] PD Mitchener, $C^{*}$-categories, Proc. London Math. Soc. (3) 84 (2002) 375-404 MR1881396

[30] PD Mitchener, Addendum to: “Coarse homology theories" [Algebr. Geom. Topol. 1 (2001), 271-297; MR1834777], Algebr. Geom. Topol. 3 (2003) 1089-1101 MR2012966

[31] PD Mitchener, $C^{*}$-categories, groupoid actions, equivariant $K K$-theory, and the Baum-Connes conjecture, J. Funct. Anal. 214 (2004) 1-39 MR2079884

[32] P D Mitchener, B Norouzizadeh, T Schick, Coarse homotopy theory, in preparation 
[33] E K Pedersen, C A Weibel, A nonconnective delooping of algebraic $K$-theory, from: "Algebraic and geometric topology (New Brunswick, N.J., 1983)", (A Ranicki, N Levitt, F Quinn, editors), Lecture Notes in Math. 1126, Springer, Berlin (1985) 166-181 MR802790

[34] J Roe, Index theory, coarse geometry, and topology of manifolds, CBMS Regional Conference Ser. in Math. 90, Conference Board of Math. Sci., Washington, DC (1996) MR1399087

[35] J Roe, Comparing analytic assembly maps, Q. J. Math. 53 (2002) 241-248 MR1909514

[36] J Roe, Lectures on coarse geometry, Univ. Lecture Series 31, Amer. Math. Soc. (2003) MR2007488

[37] F Waldhausen, Algebraic K-theory of spaces, from: "Algebraic and geometric topology (New Brunswick, N.J., 1983)”, (A Ranicki, N Levitt, F Quinn, editors), Lecture Notes in Math. 1126, Springer, Berlin (1985) 318-419 MR802796

[38] M Weiss, Excision and restriction in controlled $K$-theory, Forum Math. 14 (2002) 85-119 MR1880196

[39] M Weiss, B Williams, Assembly, from: "Novikov conjectures, index theorems and rigidity, Vol. 2 (Oberwolfach, 1993)", (S C Ferry, A Ranicki, J Rosenberg, editors), London Math. Soc. Lecture Note Ser. 227, Cambridge Univ. Press (1995) 332-352 MR1388318

[40] N Wright, $C_{0}$ coarse geometry and scalar curvature, J. Funct. Anal. 197 (2003) 469-488 MR1960422

[41] N Wright, The coarse Baum-Connes conjecture via $C_{0}$ coarse geometry, J. Funct. Anal. 220 (2005) 265-303 MR2119281

[42] G L Yu, Coarse Baum-Connes conjecture, K-Theory 9 (1995) 199-221 MR1344138

School of Mathematics and Statistics, University of Sheffield Hicks Building, Sheffield, S3 7RH, UK

P.Mitchener@sheffield.ac.uk

http://www.mitchener.staff.shef.ac.uk/

Received: 24 February 2010 Revised: 23 September 2010 